\title{
A Meta-Analysis of the Effect of Physical Activity on Depressive Symptoms: Influence of the COVID-19 Pandemic
}

\author{
Meungguk Park' \& Simon M. Pack ${ }^{2}$ \\ 'Southern Illinois University, USA \\ 2 St. John's University, New York, USA
}

\begin{abstract}
Due to the COVID-19 pandemic, a significant number of people experienced higher levels of depression. Concerning the unprecedented impact of depression on physical health, it is important to combat this situation. Physical activity (PA) has been found to have a positive effect on reducing depression (Schuch et al., 2018). However, there is a lack of a systematic review of the association between PA and depression during these unique times of the COVID-19 pandemic. This study was designed to serve as a meta-analysis of the overall effects of PA on depression in studies conducted before and during the COVID-19 pandemic.
\end{abstract}

Keywords: physical activity, depression, meta-analysis

\section{Article History}

Received 20 September 2020

Accepted 10 October 2020

Published 31 January 2021

Available online 19 February 2021

https://doi.org/10.47544/johsk.2021.2.1.27

Corresponding Author
Meungguk Park
parkm@siu.edu

\section{Methods}

Empirical studies on the link between PA and depression were searched from academic databases including PubMed and EBSCO. A total of 15 studies (total participants were 170,195) qualified for inclusion in the meta-analysis. Comprehensive Meta-Analysis version 3 was used to compute weighted average effect size and random-effects analyses were conducted.

\section{Results}

The average effect size (odds ratio) across all 15 studies was $0.722(95 \% \mathrm{Cl}=0.651,0.800, \mathrm{Z}=-6.229, \mathrm{p}<.001$ ), demonstrating that study participants with high levels of PA had lower incidence of depression, compared to those with low PA levels. For the subgroup analysis, significant effects of high PA were found in the 11 studies conducting before the COVID 19 as well as in the four studies that assessed the influence of the COVID-19 (odds ratio $=.734, p$ $<.001$; odds ratio $=.683, p<.001$ respectively). The $Q$-value $(Q=41.061, p<.001)$ and I-squared value (65.904) demonstrated that the studies were heterogeneous.

\section{Journal of Health, Sports, \& Kinesiology | ISSN 2692-9864 | www.johsk.com}


| 2021 | Volume 2 | Issue 1 | The Journal of Health, Sports, and Kinesiology |

\section{Conclusion}

The main finding of this study supported the evidence that PA can play an important role in reducing depression, especially during the COVID-19 pandemic. Public health professionals should incorporate the significance of improving PE levels into social distancing guidelines (CDC, 2020).

\section{Journal of Health, Sports, \& Kinesiology | ISSN 2692-9864 | www.johsk.com}

